EPJ Web of Conferences 76, 01018 (2014)

DOI: $10.1051 /$ epjconf/20147601018

(C) Owned by the authors, published by EDP Sciences, 2014

\title{
Concentration organic components in the hydrocarbon fuel particles conditions and characteristic of ignition
}

\author{
Vladimir Salomatov ${ }^{1}$, Semen Syrodoy $^{2}$, and Nadegda Gutareva ${ }^{2}$ \\ ${ }^{1}$ Institute of Thermophysics named after S.S. Kutateladze SB RAS, 630090 Novosibirsk, Russia \\ ${ }^{2}$ National Research Tomsk Polytechnic University, 634050 Tomsk, Russia
}

\begin{abstract}
The problem of particle ignition coal-water fuel (WCF), located in the high-flow environment has been solved numerically. The flow of the main processes of joint thermal treatment (thermal conductivity, evaporation, thermal decomposition, chemical reaction of vapor and carbon) has been taken into account. Set the scale effect of the concentration of the organic portion of the fuel on the dynamics of particle ignition of fuel. The obtained results ignition delay time values are coordinated well with published experimental data.
\end{abstract}

\section{Introduction}

Manufacturing, transportation and preparation for burning coal-water fuel (WCF) are associated with a number of technical and technological problems $[1,2]$. One such problem is the choice of concentrations of the major components (coal and water) to ensure manufacturability WCF, the minimum cost of fuelpreparation process, combustion efficiency and sustained inflammation.

Until recently, the hydrocarbon fuel is used infrequently due to the lack of a general theory that describes the whole complex of physical and chemical processes occurring in the stages of preparation and combustion. There are poorly understood processes of particle ignition WCF. One of the problems in the theory of ignition of coal-water fuel is to determine the optimal possible (on the final characteristics of combustion) of (shares directly coal and water). Experimental study of the effect of concentration on the component conditions and ignition characteristics of particles WCF is quite difficult due to problems in the registration parameters of heterogeneous fuel and ignition delay time. Therefore, most likely, the most rational is the mathematical modeling of these processes.

Purpose - to study the results of mathematical modeling of heat transfer in coal-water fuel particle concentration effect of the organic part WCF conditions and ignition characteristics of the latter.

The physical model of heat transfer in coal-water fuel has been adopted when the problem has been stated, taking into account co-occurring processes of warming due to heat conduction, evaporation of moisture vapor filtration to the heated surface of the particle in the form of a sphere, the thermal decomposition of coal to gaseous and solid reaction products, filtering volatile particles to the surface, chemical interaction of water vapor with carbon coke oxidation of volatile oxygen ambient, supply heat to the particle surface due to convection, heat conduction and radiation [3].

This is an Open Access article distributed under the terms of the Creative Commons Attribution License 4.0, which permits unrestricted use, distribution, and reproduction in any medium, provided the original work is properly cited. 


\section{EPJ Web of Conferences}

The system of differential equations with the appropriate initial and boundary conditions has the following form:

Energy equation for the initial (wet) of the fuel:

$$
\begin{gathered}
C_{1} \rho_{1} \frac{\partial T_{1}(r, t)}{\partial t}=\lambda_{1} \cdot\left[\frac{\partial^{2} T_{1}(r, t)}{\partial r^{2}}+\frac{2}{r} \cdot \frac{\partial T_{1}(r, t)}{\partial r}\right] \\
t>0,0<r<r_{V}, T<T_{V} ;
\end{gathered}
$$

Energy equation for "dehydrated" part WCF:

$$
\begin{gathered}
C_{2} \rho_{2} \frac{\partial T_{2}(r, t)}{\partial t}=\lambda_{2} \cdot\left[\frac{\partial^{2} T_{2}(r, t)}{\partial r^{2}}+\frac{2}{r} \cdot \frac{\partial T_{2}(r, t)}{\partial r}\right]-\sum Q_{i} \cdot W_{i}-u_{s} C p_{s} \cdot \rho_{s} \frac{\partial T_{2}(r, T)}{\partial r} \\
t>0, r_{V}<r<r_{0}, T \geq T_{V} ;
\end{gathered}
$$

The equation of chemical kinetics for the organic portion of the fuel:

$$
\begin{gathered}
\frac{\partial \eta_{2}(r, t)}{\partial t}=(1-\eta(r, t)) \cdot k_{0} \cdot \rho \cdot \exp \left(-\frac{E}{R \cdot T_{1}(r, t)}\right) \\
t>0, r_{V}<r<r_{0}, T \geq T_{V} ;
\end{gathered}
$$

Filtration equation for water vapor and gaseous products of thermal decomposition:

$$
\begin{gathered}
\frac{\partial p_{s}(r, t)}{\partial t}=\frac{m \cdot \xi \cdot Z}{K_{P}}\left(\frac{\partial p_{s}^{2}(r, t)}{\partial r^{2}}+\frac{2}{r} \frac{\partial p_{s}(r, t)}{\partial r}\right) \\
t>0, r_{V}<r<r_{0}, T \geq T_{V} ;
\end{gathered}
$$

At the interface of the "original WCF — dry coal" there is the boundary condition of the 4th kind:

$$
\begin{gathered}
\left.\lambda_{1} \frac{\partial T_{1}\left(r_{V}, t\right)}{\partial r}\right|_{r=r_{V}-0}-\left.\lambda_{2} \frac{\partial T_{2}\left(r_{V}, t\right)}{\partial r}\right|_{r=r_{V}+0}=Q_{V} \cdot W_{V} ; \\
T_{1}\left(r_{V} ; t\right)=T_{2}\left(r_{V} ; t\right)=T_{V} .
\end{gathered}
$$

The system of equations (1-4) has been solved with the following boundary conditions and closing ratios: $0<r<r_{0} ; T(r, 0)=T_{0} ; P(r, 0)=P_{0} ; \eta(r, 0)=\eta_{0}$;

$$
\begin{gathered}
-\lambda_{2} \frac{\partial T_{1}\left(r_{0}, t\right)}{\partial r}=\alpha \cdot\left[T_{c}-T_{1}\left(r_{0}, t\right)\right]+\varepsilon \cdot \sigma \cdot\left[T_{c}^{4}-T_{1}^{4}\left(r_{0}, t\right)\right]+\sum_{i} Q_{i} \cdot W_{i} \\
\frac{\partial T_{2}(0, t)}{\partial r}=0 \\
\frac{\partial P\left(r_{V}, t\right)}{\partial r}=\frac{K_{P}}{v} u\left(r_{V} ; t\right) \\
p\left(r_{0} ; t\right)=p_{0} . \\
01018-\text { p.2 }
\end{gathered}
$$


The rate of reaction of $\mathrm{C}+\mathrm{H}_{2}=\mathrm{CO}+\mathrm{H}_{2}-118.485 \mathrm{MJ}$ has been calculated from the following expression:

$$
W_{i}=k_{i} \cdot c_{i} \cdot \rho_{i} \cdot \exp \left(-\frac{E}{R \cdot T(r, t)}\right) .
$$

Pyrolysis rate has been calculated using the following equation:

$$
W_{i}=(1-\eta(r, t)) \cdot k_{i} \cdot \rho_{i} \cdot \exp \left(-\frac{E}{R \cdot T(r, t)}\right) .
$$

Ignition of WCF - heterogeneous process defined by ignition kinetics of thermal decomposition products, and the reaction of water vapor with carbon. There are following reactions on the surface of the particles:

1. $\mathrm{H}_{2}+0.50_{2}=\mathrm{H}_{2} \mathrm{O}+141900 \frac{\mathrm{kj}}{\mathrm{kg}}$.

2. $\mathrm{CO}+0.50_{2}=\mathrm{CO}_{2}+10090 \frac{\mathrm{kj}}{\mathrm{kg}}$.

3. $\mathrm{CH}_{4}+\mathrm{O}_{2}=\mathrm{CO}_{2}+\mathrm{H}_{2}+55546 \frac{\mathrm{kj}}{\mathrm{kg}}$.

Dependence of these reactions has been used to determine the velocity:

$$
W_{i}=k_{i} \cdot c_{i} \cdot \rho_{i} \cdot \exp \left(-\frac{E}{R \cdot T\left(r_{0}, t\right)}\right)
$$

$\rho$-density gaseous components has been determined from the equation of state:

$$
\rho_{i}=\frac{p(r, t) \cdot \mu}{T\left(r_{0}, t\right) \cdot R} .
$$

Filtration rate has been determined by a pair of Darcy's law:

$$
u=-\frac{K_{P}}{v} \frac{\partial p}{\partial r} .
$$

There are the following notations: the initial temperature of $\mathrm{T}_{0}-$ particles, $\mathrm{K} ; \mathrm{T}_{\mathrm{c}}$ - ambient temperature, $\mathrm{K} ; r_{\mathrm{U}}$ - radius evaporation front, $\mathrm{m} ; r_{0}$ - outer radius of the particle, $\mathrm{m} ; \sigma$ - constant blackbody radiation, $\frac{W}{m^{2} K^{4}} ; \alpha$-convective heat transfer coefficient, $\frac{W}{m^{2} K}, \lambda_{1}$ thermal conductivity of the original WCF, $\frac{W}{m \cdot K}$; $\lambda_{2}-$ dry thermal conductivity of the WCF, $\frac{W}{\mathrm{~m} \cdot K} 1 ; \mathrm{C}_{1}$ - heat capacity of the initial part of WCF, $\frac{j}{\mathrm{~kg} \cdot K}$; $\mathrm{C}_{2}$ - is the thermal conductivity of the dry WCF, $\frac{j}{\mathrm{~kg} \cdot \mathrm{K}} ; W_{v}$ - mass evaporation rate of water, $\frac{\mathrm{kg}}{\mathrm{m}^{2} \cdot \mathrm{s}}$; $Q_{v}$ - thermal effect of the evaporation of water $\frac{j}{\mathrm{~kg}} ; Q_{i}$ thermal effect of the chemical reaction, $\frac{j}{\mathrm{~kg}}$; $k_{i}$ - pre-exponential factor of the chemical reaction, $\frac{1}{s} ; E_{i}$ - activation energy of a chemical reaction; $\frac{j}{m o l} ; u_{s}$ - filtration rate of water vapor, $\frac{\mathrm{m}}{s} ; c p_{s}$ - isobaric heat capacity of water vapor, $\frac{j}{\mathrm{~kg} \cdot \mathrm{K}} ; \rho_{s}-$ vapor density, $\frac{\mathrm{kg}}{\mathrm{m}^{3}} ; p_{s}$ - vapor pressure in $\mathrm{Pa} ; \mathrm{m}$ - porosity; $\mathrm{Z}$ - degree of compressibility of water vapor; $K_{P}$ - permeability porous structure, $\mathrm{m}^{2} ; \mu$ - molar mass of water, $\frac{\mathrm{kg}}{\mathrm{mol}} ; v$ - kinematic viscosity of water vapor, $\frac{m^{2}}{s} ; C_{1}$ - concentration of the respective component of the chemical reaction; i - ordinal chemical reaction component.

Coordinate border of evaporating has been found from the expression:

$$
r_{F}=r_{0}-\int u_{F} \cdot d \tau .
$$




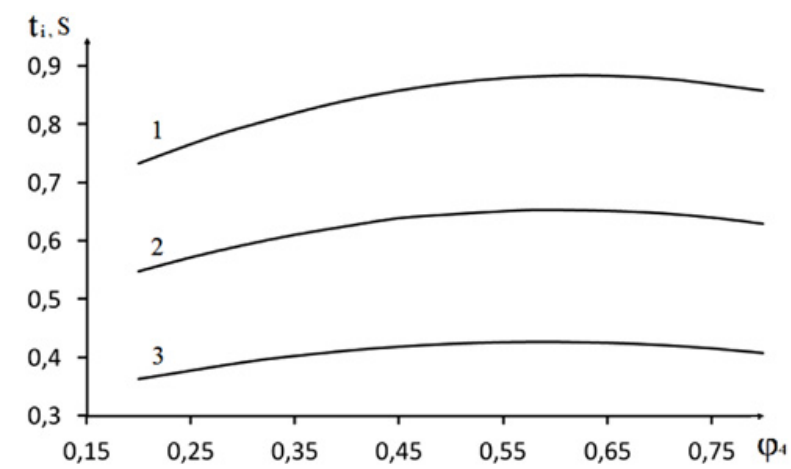

Figure 1. The ignition delay time (td) depending on the mass fraction of carbon in the particle diameter of component (d): $1-0.8 \cdot 10^{-3} \mathrm{~m} ; 2-0.6 \cdot 10^{-3} \mathrm{~m} ; 3-0.4 \cdot 10^{-3} \mathrm{~m}$.

Where: $u_{F}=\frac{W_{v}}{\rho}$-linear speed of advance of the evaporation front, $\frac{m}{s}$.

Mass evaporation rate has been calculated from the expression:

$$
W_{V}=W_{0} \cdot \exp \left(\frac{Q_{V} \cdot \mu \cdot\left(T_{1}-T_{0}\right)}{R \cdot T_{0} \cdot T_{1}}\right) .
$$

Where: $T_{1}$ - evaporation temperature at the boundary, $\mathrm{K} ; T_{0}$ - fluid temperature corresponding to the freezing point, $\mathrm{K} ; W_{0}$ - rate of evaporation at temperature $T_{0}, \frac{\mathrm{kg}}{\mathrm{m}^{2} \cdot \mathrm{s}} ; \mu$ - molar mass of water vapor, $\frac{\mathrm{kg}}{\mathrm{mol}}$; $\mathrm{R}$ - universal gas constant, $\frac{j}{\mathrm{~mol} \cdot \mathrm{k}}$.

The system of nonlinear Eqs. (1) - (15) has been solved by finite difference method using the developed for solving heat and mass transfer under intensive phase transitions $[4,5]$ and pyrolysis of composite polymeric materials [6] iterative algorithm. Selection steps of the difference grid in time and the spatial coordinate has been analogous to [7,8] in connection with the rapid heating and high temperature gradient. Reliability of the results of numerical modeling of the investigated processes has been controlled test of conservative difference schemes by the method developed for the analysis of the results of solving nonlinear problems of ignition and combustion of condensed substances $[9,10]$.

The anthracite deposit of the Novosibirsk region has been selected as the base material for Leafage coal-water fuel.

Thermo-physical properties of wet and "dehydrated" portion of the fuel have been calculated the volume fractions of the components:

$$
\begin{aligned}
& \lambda_{1}=\varphi_{3} \lambda_{3}+\varphi_{4} \lambda_{4} \\
& \lambda_{2}=\varphi_{4} \lambda_{4}+\varphi_{5} \lambda_{5} .
\end{aligned}
$$

Where $\varphi$ - volume fraction of the corresponding component of fuel: 3 - water; 4 - carbon; 5 - air.

The dependence of the ignition delay $t_{i}$ concentration (mass fraction) of coal $\left(\varphi_{4}\right)$ WCF at different fuel particle diameters are given in Fig. 1, obtained by numerical simulation. It is seen that with increasing $\varphi_{4}$ increases to values $\mathrm{t}_{i} \varphi 4 \sim 0.55-0.65$. Then increase the share of coal leads to some relative $(5-10 \%)$ reduction in the ignition delay time. The obtained dependences $t_{i}\left(\varphi_{4}\right)$ can be explained by a change in the effective thermal conductivity WCF to change in water. The thermal conductivity of the latter is significantly lower $\lambda$ coal components. Accordingly, the heating of a thin surface layer of coal-water fuel and the boiling temperature of water at high concentration liquid is faster in comparison with a homogeneous charcoal. 


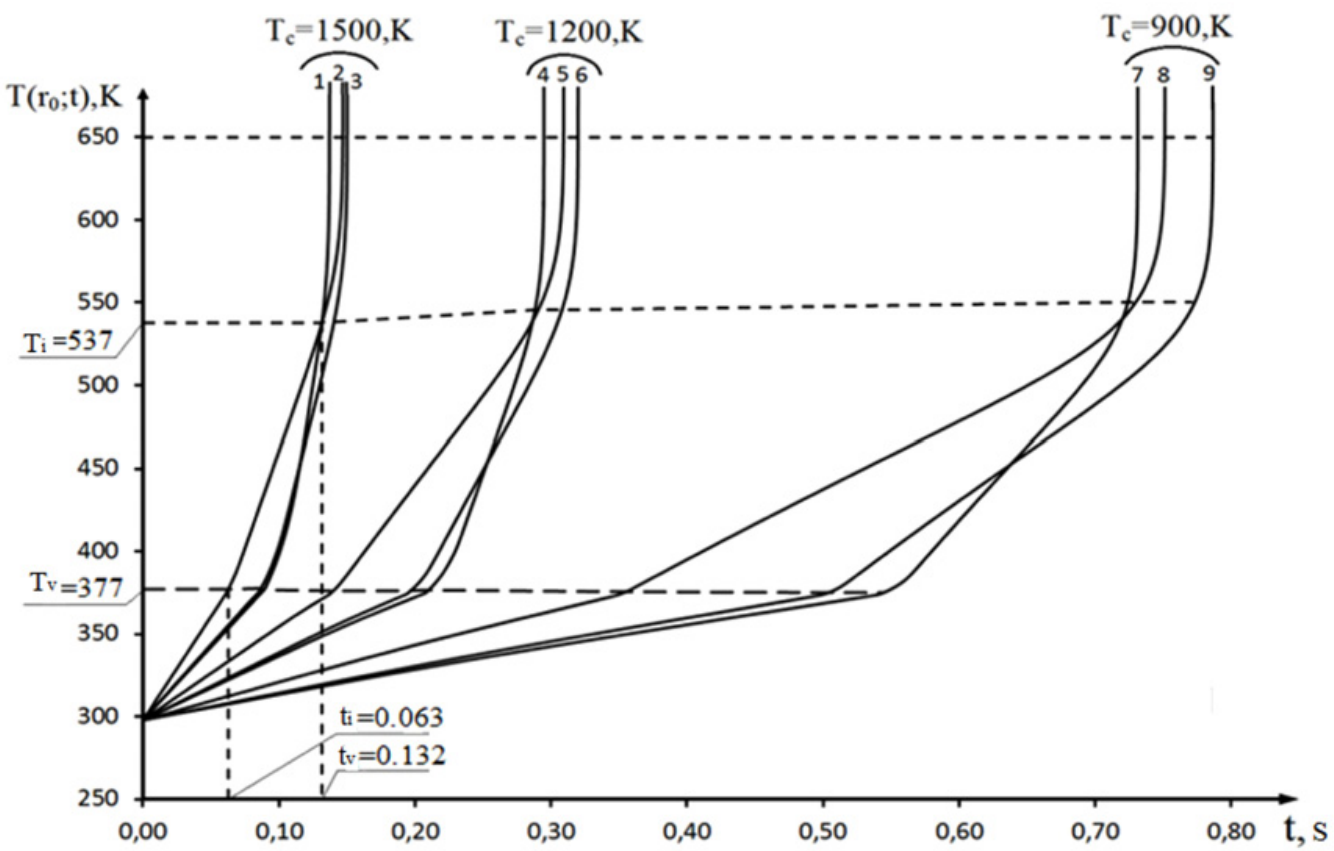

Figure 2. The surface temperature of the particle $\mathrm{T}(\mathrm{r} 0$; $\mathrm{t})$ from the time the entire process of ignition $\mathrm{t}$, particle diameter $\mathrm{d}=0.4 \cdot 10^{-3} \mathrm{~m}$ in a medium with a temperature $\mathrm{Tc}$, the shared content with different components in the coal WCF: $1-\varphi_{4}=0.3 ; 2-\varphi_{4}=0.8 ; 3-\varphi_{4}=0.5 ; 4-\varphi_{4}=0.3 ; 5-\varphi_{4}=0.8 ; 6-\varphi_{4}=0.5 ; 7-\varphi_{4}=0.3 ; 8-\varphi_{4}=0.8$; $9-\varphi_{4}=0.5$.

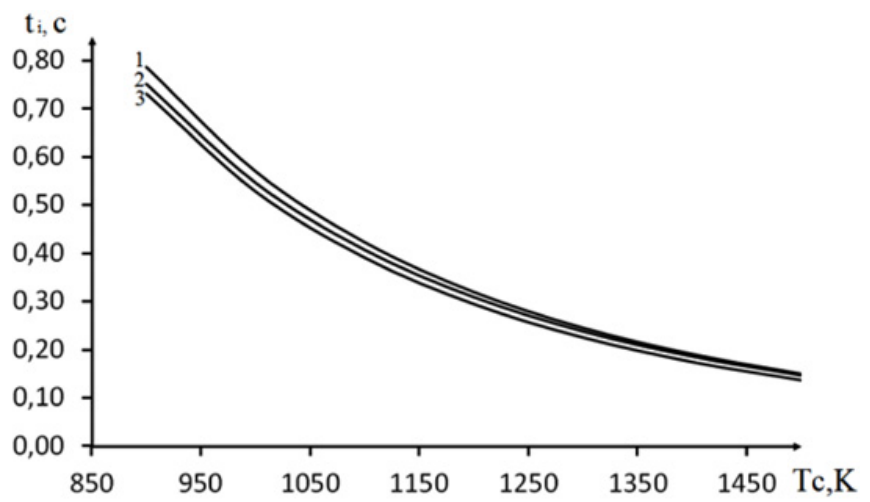

Figure 3. The dependence of the ignition delay time on the external temperature $\mathrm{Tc}$ for a particle diameter $\mathrm{d}=0.4 \cdot 10^{-3} \mathrm{~m}$ with the following content of the coal share: $1-\varphi_{4}=0.5 ; 2-\varphi_{4}=0.8 ; 3-\varphi_{4}=0.3$.

A slight decrease in the values of $t_{i}$ (within 5-6\%) at low water contents (less than 0.3 by weight) is probably due to a decrease in the cost of heat on the phase transformation (evaporation). But on the whole range of variation of $t_{i}$ at $0,2 \leq \varphi_{4} \leq 0,8$ is relatively small (less than $35 \%$ ).

Figure 2 shows the temperature dependence of the particle's surface coal-water fuel from time to time the ignition WCF for three values of ambient temperature. On each curve can be divided into three characteristic regions. The first (up to $t=t_{w}$ ) corresponds to an interval time of heating to the boiling point of the particle (or close thereto), and it is complete "dewatering". Slope of the curve is determined 


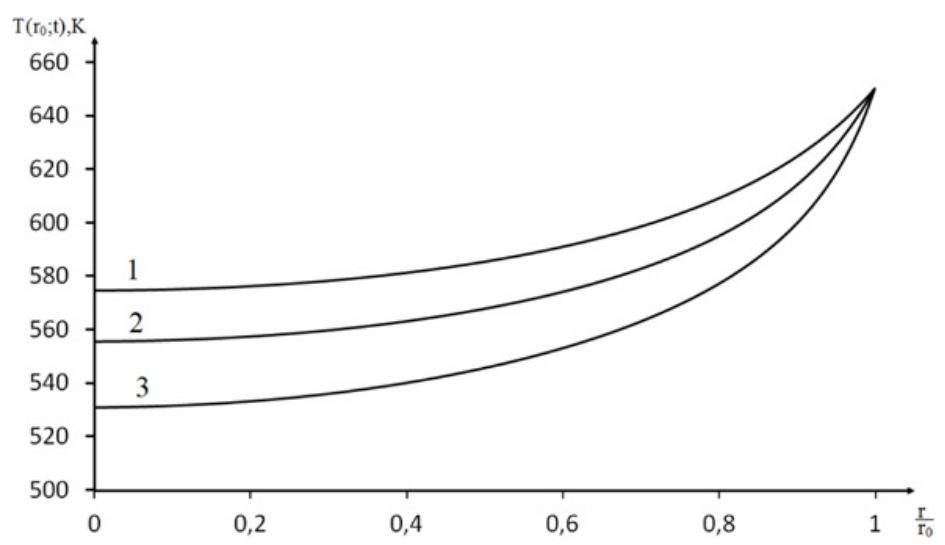

Figure 4. The temperature distribution along the radius of the particle diameter $\mathrm{d}=0.4 \cdot 10^{-3} \mathrm{~m}$ at the time of ignition, when the content of coal: $1-\varphi_{4}=0.8 ; 2-\varphi_{4}=0.5 ; 3-\varphi_{4}=0.3$.

by the thermal conductivity of coal moisture saturation. The second portion ( $u p$ to $t=t_{i}$ ) corresponds to the period of warm and dry thermal decomposition of coal.

In this state, the thermal conductivity of the latter is substantially less (as compared to the first portion). Accordingly, the temperature of the surface grows faster than the first portion. At $t=t_{i}$ condition WCF ignition and further temperature rises to values corresponding to the combustion of coal-water fuel.

Depending on the ignition delay time on the ambient temperature for a typical particle diameter $0,4 \cdot 10^{-3} \mathrm{~m}$ and three values of mass fractions of organic components shown in Fig. 3. Type dependencies are generally quite good agreement with the modern theory of ignition of condensed substances. Temperature distributions along the radius of the particle at the moment of ignition WCF at different $\varphi_{4}$ show (Fig. 4) that the difference $\mathrm{T}$ across the entire thickness of fuel does not exceed $120 \mathrm{~K}$. In other words, it may be concluded that ignition occurs after the complete evaporation of all the moisture contained in the porous structure WCF, but low enough temperatures the particle surface.

The results obtained suggest the possibility of varying the mass fraction of component (water and coal directly) over a wide range without changing significantly conditions and the ignition characteristics of small particle size of coal-water fuel.

\section{References}

[1] G. Khodakov J. Therm. Eng. Vol. 54. No. 1, 2007. p. 36-77

[2] A. Belov Burning high watered fuel in the form of coal-water slurries. (Sciences, p. 103-111. 1967)

[3] S. Syrodoy, V Salomatov and G. Kuznetsov, Polzunovskii Gaz. V. 4. No. 3. 2013. p. 28-33

[4] G. Kuznetsov, P. Strizhak. J. Eng. Therm. V. 17. No 3. 2008. p. 244-252

[5] G. Kuznetsov, P. Strizhak. J. Eng. Therm. V. 18. No 1. 2009. p. 72-79

[6] V. Strakhov, A. Garashchenko, G.Kuznetsov and V. Rudzinskii. J. Com. Exp. Sh.W. V. 37, No 2. 2001. p.178-186

[7] G. Kuznetsov, G. Mamontov and G. Taratushkina J. Com. Exp. Sh.W. V 40. No 1. 2004. p.70-76

[8] G. Kuznetsov, G. Mamontov and G. Taratushkin, Rus. J. Phys. Chem.B. V. 23, No 5. 2004. p. $62-68$

[9] G. Kuznetsov, P. Strizhak. Int. J. Heat Mass Transfer. V. 53. No 5-6. 2010. p. 923-930

[10] G. Kuznetsov, P. Strizhak. J. Eng. Therm. V. 18. No 2. 2009. p. 162-167 